\title{
Capillary electrophoresis-mass spectrometry using noncovalently coated capillaries for the analysis of biopharmaceuticals
}

\author{
R. Haselberg • V. Brinks • A. Hawe • G. J. de Jong • \\ G. W. Somsen
}

Received: 4 November 2010 /Revised: 24 December 2010 /Accepted: 27 January 2011 /Published online: 14 February 2011

(C) The Author(s) 2011. This article is published with open access at Springerlink.com

\begin{abstract}
In this work, the usefulness of capillary electrophoresis-electrospray ionization time-of-flight-mass spectrometry for the analysis of biopharmaceuticals was studied. Noncovalently bound capillary coatings consisting of Polybrene-poly(vinyl sulfonic acid) or Polybrene-dextran sulfate-Polybrene were used to minimize protein and peptide adsorption, and achieve good separation efficiencies. The potential of the capillary electrophoresis-mass spectrometry (CE-MS) system to characterize degradation products was investigated by analyzing samples of the drugs, recombinant human growth hormone (rhGH) and oxytocin, which had been subjected to prolonged storage, heat exposure, and/or different $\mathrm{pH}$ values. Modifications could be assigned based on accurate masses as obtained with time-of-flight-mass spectrometry (TOF-MS) and migration times with respect to the parent compound. For heat-exposed rhGH, oxidations, sulfonate formation, and deamidations were observed. Oxytocin showed strong deamidation (up to $40 \%$ ) upon heat exposure at low $\mathrm{pH}$, whereas at medium and high $\mathrm{pH}$, mainly dimer $(>10 \%)$ and trisulfide formation (6-7\%) occurred. Recombinant human interferon- $\beta-1 \mathrm{a}(\operatorname{rhIFN}-\beta)$ was used to evaluate the capability of the CE-MS method to assess glycan heterogeneity of pharmaceutical proteins. Analysis of this $N$ -
\end{abstract}

R. Haselberg $(\bowtie) \cdot$ G. J. de Jong · G. W. Somsen Department of Biomedical Analysis, Utrecht University, PO Box 80082, 3508 TB Utrecht, The Netherlands e-mail: r.haselberg@uu.nl

V. Brinks

Department of Pharmaceutics, Utrecht University, PO Box 80082, 3508 TB Utrecht, The Netherlands

A. Hawe

Division of Drug Delivery Technology, Leiden/Amsterdam Center for Drug Research, Leiden University,

PO Box 9502, 2300 RA Leiden, The Netherlands glycosylated protein revealed a cluster of resolved peaks which appeared to be caused by at least ten glycoforms differing merely in sialic acid and hexose $N$-acetylhexosamine composition. Based on the relative peak area (assuming an equimolar response per glycoform), a quantitative profile could be derived with the disialytated biantennary glycoform as most abundant (52\%). Such a profile may be useful for in-process and quality control of rhIFN- $\beta$ batches. It is concluded that the separation power provided by combined capillary electrophoresis and TOF-MS allows discrimination of highly related protein species.

Keywords Biopharmaceuticals · Capillary electrophoresis . Electrospray ionization $\cdot$ Mass spectrometry $\cdot$ Noncovalent coatings

\section{Introduction}

With efficient methodologies available in biotechnology today, increasing numbers of recombinantly manufactured pharmaceutical peptides and proteins are being commercialized $[1,2]$. The assessment of biopharmaceutical quality in terms of identity, content, and purity is an important issue during manufacturing. The biotechnological production process may show variability, which can introduce product diversity, isoforms, and closely related degradation products [3, 4]. With the appearance of biosimilars, i.e., attempted copies of the original biopharmaceutical [4], the demand for extensive characterization of therapeutic proteins and peptides has even further increased. The variability and stability between biosimilar and originator product might differ even more significantly than between batches of the same product. Clearly, there is an increasing demand for suitable analytical methods that allow not only protein 
and peptide identification, but also the separation and quantification of impurities and possible degradation products.

Capillary electrophoresis-mass spectrometry (CE-MS) provides the high separation efficiency and mass-selective detection $[5,6]$ that can be very useful for biopharmaceutical product characterization. Many protein modifications, like glycosylation and deamidation, may involve changes of net charge of a protein and, thus, also of its electrophoretic mobility. As capillary electrophoresis (CE) has the intrinsic capacity to produce narrow peaks, it shows good potential for the separation of a variety of protein modifications in a single run. Mass spectrometry (MS) detection of high mass accuracy and resolution, such as provided by time-of-flight (TOF) instruments can yield accurate information on the molecular weight of analyzed proteins species. CE-MS is most commonly carried out applying electrospray ionization (ESI) using a sheath liquid interface, which is relatively robust and easy to implement. The sheath liquid composition and flow rate can be optimized to enhance protein signal intensities $[7,8]$. So far, the application of sheath liquid CE-MS for the analysis of biopharmaceuticals has been quite limited. Staub et al. developed a CE-TOF-MS method to distinguish natural human growth hormone (hGH) from recombinant hGH [9], and Catai et al. showed the feasibility of CE-ion trap-MS for the determination of some degradation products in hGH after heat exposure and prolonged storage [10, 11]. Neusüß et al. and Sanz-Nebot et al. demonstrated the usefulness of CE-TOF-MS for the glycoform characterization of erythropoietin [12-16].

The tendency of protein molecules to adsorb to fused-silica may result in deterioration of CE separation efficiencies. In order to prevent adverse interactions, it is a common strategy to coat the internal wall surfaces with agents that minimize protein adsorption $[17,18]$. Two general coating strategies can be discerned in CE: dynamic and static coating [19]. Dynamic coating agents are continuously present in the BGE and reduce protein adsorption by reversibly binding to adsorption sites on the silica surface. For CE-MS, a disadvantage of this type of coating is that coating agents may enter the ion source and, thereby, can result in ionization suppression of the analyte and contamination of the mass spectrometer. In order to prevent MS detection interferences, static coatings have to be used. These coatings are permanently attached to the capillary surface by adsorption or by covalent coating involving silane chemistry and polymerization schemes. The former capillary coatings can be produced simply by flushing the capillary with solutions of highly adsorptive agents. Very stable and uniform adsorbed coatings can be produced by applying multiple layers of oppositely charged polymers, as indicated for the first time by Katayama et al. [20, 21] and demonstrated by others subsequently [22-29].
Over the last years, we have studied and shown the usefulness of a bilayer coating of Polybrene-poly(vinyl sulfonic acid) (PB-PVS) and a triple layer coating of Polybrene-dextran sulfate-Polybrene (PB-DS-PB) for the CE-UV and CE-MS analysis of peptides and proteins $[10,11,30-33]$. The negatively charged PB-PVS coating was used in combination with a background electrolyte (BGE) of medium or high $\mathrm{pH}$ for the analysis of acidic peptides and proteins $[10,11,30,31]$, whereas for basic proteins, the positively charged PB-DS-PB coating was used in combination with a low-pH BGE $[32,33]$. The multilayer coatings exhibit a pH-independent and highly reproducible EOF and effectively prevent protein adsorption [34], resulting in efficient protein separations. In addition, these coatings show very good compatibility with electrospray ionization-mass spectrometry (ESI-MS) detection [31, 32]. As a result, both CE-MS systems showed good performance for intact protein analysis yielding RSDs for migration time and peak area within $2 \%$ and $10 \%$, respectively $[10,11,32,33]$. Linear relationships between protein concentration and peak area were achieved, and detection limits as low as $11 \mathrm{nM}$ could be obtained [32].

In the study presented here, the performance and usefulness of CE-MS systems employing PB-PVS and PB-DS-PB coated capillaries for the characterization of biopharmaceuticals was investigated. Representative samples of the drugs, recombinant human growth hormone (rhGH, $22 \mathrm{kDa}, p I$ 5.1), recombinant human interferon- $\beta$ 1a (rhIFN- $\beta, 23 \mathrm{kDa}, p I$ 9.6), and oxytocin (1 kDa, $p I$ 8.6) were used for testing. The potential of the CE-MS systems to separate and identify degradation products resulting from heat stress and/or prolonged storage of rhGH and oxytocin was studied. In addition, rhIFN- $\beta$ was analyzed to evaluate the capability of the PB-DS-PB CE-TOF-MS method to assess the glycoform heterogeneity of a pharmaceutical protein. Some attention was paid to optimization of separation and detection conditions, but emphasis was on the type of information on the sample composition that can be obtained with CE-TOF-MS.

\section{Materials and methods}

\section{Chemicals}

Polybrene (hexadimethrine bromide, PB; average $\mathrm{Mw}$ $15,000)$, dextran sulfate (DS; average $\mathrm{Mw}>500,000$ ) sodium salt, $25 \%(w / v)$ poly (vinyl sulfonic acid) (PVS; average Mw 4,000-6,000), isopropanol, and acetonitrile were purchased from Sigma Aldrich (Steinheim, Germany). Acetic acid, formic acid, and 25\% (v/v) ammonium hydroxide were obtained from Merck (Darmstadt, Germany). The ammonium 
formate BGE was made by diluting ammonium hydroxide to $75 \mathrm{mM}$ and adjusting the $\mathrm{pH}$ to 8.5 with $1 \%(v / v)$ formic acid in deionized water. Acetic acid BGEs were prepared by diluting acetic acid to the desired concentration with deionized water and adjusted to the appropriate $\mathrm{pH}$ with $1 \%(v / v)$ ammonium hydroxide in deionized water.

A solution of rhGH had been prepared previously by dissolving somatropin CRS (European Directorate for the Quality of Medicines, Strasbourg, France) in $30 \mathrm{mM}$ Tris phosphate $(\mathrm{pH} 7.5)$ to a concentration of $1.5 \mathrm{mg} / \mathrm{mL}$. An aliquot of this sample was incubated at $40^{\circ} \mathrm{C}$ for $24 \mathrm{~h}$ and subsequently had been stored for 12 months at $-18^{\circ} \mathrm{C}$. Oxytocin acetate powder (Sigma Aldrich, Zwijndrecht, The Netherlands) had been dissolved previously in $50 \mathrm{mM}$ phosphate buffer with a $\mathrm{pH}$ of $2.0,4.5,7.0$, and 9.0 to a final concentration of $50 \mu \mathrm{g} / \mathrm{mL}$. Aliquots of these solutions had been subsequently exposed to $70^{\circ} \mathrm{C}$ for $64 \mathrm{~h}$ and then were stored at $4{ }^{\circ} \mathrm{C}$ until analysis. Bulk rhIFN- $\beta$ 1a was supplied by Biogen Idec Inc. (Cambridge, MA, USA) as a $0.27 \mathrm{mg} / \mathrm{ml}$ solution in $100 \mathrm{mM}$ sodium phosphate buffer and $200 \mathrm{mM}$ sodium chloride at $\mathrm{pH}$ 7.2. Reformulated rhIFN $\beta-1 \mathrm{a}$ was produced by dialysis of bulk rhIFN- $\beta$-1a with a $3.5-\mathrm{kDa}$ molecular weight cut-off (MWCO) Slide-A-Lyzer Cassette (Perbio Science, Etten-Leur, The Netherlands) against a commercially used formulation containing $20 \mathrm{mM}$ sodium acetate buffer, $150 \mathrm{mM}$ L-arginine monohydrochloride and $0.04 \mathrm{mM}$ Tween 20 (Sigma Aldrich, Zwijndrecht, The Netherlands) at $\mathrm{pH} 4.8$ and subsequent filtration through a $0.22-\mu \mathrm{m}$ polyethersulfone membrane (Millipore, Amsterdam, The Netherlands). This solution was stored at $4{ }^{\circ} \mathrm{C}$ until analysis. Prior to $\mathrm{CE}-\mathrm{MS}$ analysis, the formulation buffer was exchanged for water using a MWCO filter of $3 \mathrm{kDa}$. The final solution had a rhIFN- $\beta$ concentration of $450 \mu \mathrm{g} / \mathrm{mL}$.

\section{CE system}

The experiments were carried out on a P/ACE MDQ capillary electrophoresis instrument (Beckman Coulter, Brea, CA, USA). Fused-silica capillaries were from Polymicro Technologies (Phoenix, AZ, USA) having a total length of $80 \mathrm{~cm}$ and an internal diameter of $50 \mu \mathrm{m}$. Hydrodynamic injections were performed at 1 psi for $12 \mathrm{~s}$ (i.e., $1 \%$ of the total capillary volume). The separation voltage was $30 \mathrm{kV}$ (PB-PVS coating) or $-30 \mathrm{kV}$ (PB-DSPB coating), and the capillary temperature was $20^{\circ} \mathrm{C}$. New fused-silica capillaries were rinsed with $1 \mathrm{M} \mathrm{NaOH}$ for $30 \mathrm{~min}$ at $20 \mathrm{psi}$ and water for $15 \mathrm{~min}$ at $20 \mathrm{psi}$. After this treatment, capillaries were coated with either a PB-PVS coating or a PB-DS-PB coating using the procedures described below.
Capillary coating

PB was dissolved in deionized water to a final concentration of $10 \%(w / v)$, and DS was dissolved to $0.5 \%(w / v)$ with deionized water. PVS was diluted in deionized water to $1 \%$ $(w / v)$. The coating agents were filtered over a $0.45-\mu \mathrm{m}$ filter type HA (Millipore, Molsheim, France) prior to use. The PB-PVS coating was applied by subsequently rinsing 30 min with $10 \%(w / v)$ PB solution at 5 psi, 10 min with deionized water at $10 \mathrm{psi}, 30 \mathrm{~min}$ with $1 \%(v / v)$ PVS solution at $5 \mathrm{psi}$, and $10 \mathrm{~min}$ with deionized water at 10 psi. The PB-DS-PB coating was applied by subsequently rinsing 30 min with $10 \%(w / v)$ PB solution at $5 \mathrm{psi}, 10 \mathrm{~min}$ with deionized water at $10 \mathrm{psi}, 45 \mathrm{~min}$ with $0.5 \%(w / v)$ DS solution at 5 psi, 10 min with deionized water at $10 \mathrm{psi}, 30 \mathrm{~min}$ with $10 \%(w / v)$ PB solution at 5 psi. After the final coating agent flushing step, the capillary was rinsed 10 min with deionized water at 10 psi. The capillary was then ready for $\mathrm{CE}$ analysis with the BGE of choice. Between runs, coated capillaries were flushed with BGE for $3 \mathrm{~min}$ at 10 psi. Overnight, capillaries were filled with BGE, and tips were immersed in vials with BGE.

\section{Mass spectrometry}

MS was performed using a micrOTOF orthogonalaccelerated TOF mass spectrometer (Bruker Daltonics, Bremen, Germany). Source and transfer parameters were optimized by direct infusion of an ESI tuning mix (Agilent Technologies, Waldbronn, Germany). CE-MS coupling was realized by a co-axial sheath liquid interface (Agilent Technologies, Waldbronn, Germany). For the analysis of $\mathrm{rhGH}$, the sheath liquid was acetonitrile-water-formic acid $(75 / 25 / 5, v / v / v)$ at a flow rate of $4 \mu \mathrm{L} / \mathrm{min}$. Sheath liquids (2 $\mu \mathrm{L} / \mathrm{min}$ ) for oxytocin and rhIFN- $\beta$ analyses were isopropanol-water-acetic acid $(75 / 25 / 0.1, v / v / v)$ and isopropanol-water-acetic acid $(25 / 75 / 0.5, v / v / v)$, respectively. Sheath liquid was delivered by a $2.5-\mathrm{mL}$ gas-tight syringe (Hamilton, Reno, NV, USA) using a syringe pump of ColeParmer (Vernon Hill, IL, USA). The following optimized spray conditions were used: dry gas temperature, $180^{\circ} \mathrm{C}$; nitrogen flow, $4 \mathrm{~L} / \mathrm{min}$; and nebulizer pressure, 0.4 bar. Electrospray in positive ionization mode was achieved using an ESI voltage of $-4.5 \mathrm{kV}$. To assure proper ion transfer, the analysis of $\mathrm{rhGH}$ and $\mathrm{rh}$ IFN- $\beta$ were performed with a capillary exit and skimmer voltage of 250 and $83 \mathrm{~V}$, respectively. For the analysis of oxytocin, these voltages were set at to 200 and $50 \mathrm{~V}$, respectively. CE-MS data were analyzed using Bruker Daltonics Data Analysis software. Molecular weight determinations of proteins were performed using the "Charge Deconvolution" utility of the DataAnalysis software. 


\section{Results and discussion}

Recombinant human growth hormone

rhGH is a therapeutic protein used in the treatment of retarded growth and dwarfism caused by the inadequate production of the hormone during the growth period. The monograph of rhGH in the European Pharmacopoeia (Ph. Eur.) includes a CE method using bare fused-silica capillaries for detection of charge variants [35]. Catai et al. proposed the use of PB-PVS coated capillaries for rhGH analysis by $\mathrm{CE}$ in order to improve the method's repeatability, resolution, and efficiency [11]. Furthermore, an MS-compatible method was developed using a volatile BGE of ammonium formate [10, 11]. However, the resolution and mass accuracy of the used ion trap mass spectrometer were not sufficient to unambiguously assign rhGH degradation products. Here, we study the potential of CE-TOF-MS with PB-PVS coated capillaries for the characterization of a heat-exposed and stored $\mathrm{rhGH}$ sample.

First, a solution of $1.5 \mathrm{mg} / \mathrm{mL}$ rhGH (somatropin CRS) was analyzed by CE-TOF-MS using a BGE of 75 ammonium formate ( $\mathrm{pH} 8.5$ ). Under these conditions, both the protein ( $p I$ 5.1) and the PB-PVS coated capillary wall are negatively charged, preventing protein adsorption. A sheath liquid of low $\mathrm{pH}$ was used to allow efficient positive ESI. A narrow symmetric peak (N, 105,000) was obtained for rhGH at a migration time of approximately $11.5 \mathrm{~min}$ (Fig. 1a, peak 0). Deconvolution of the mass spectrum obtained in the apex of the peak (Fig. 1b) revealed a protein molecular weight of $22123.9 \mathrm{Da}$, which well agrees with the expected molecular weight for rhGH. Remarkably, the mass spectrum seems to comprise two charge state distributions. This might be explained by partial unfolding of the protein during ESI, which leads to increased charging and a shift of the distribution to lower $m / z$ values [36]. Next to $\mathrm{hGH}$, a small additional peak at $10.2 \mathrm{~min}$ was detected. Its mass spectrum shows major signals at $\mathrm{m} / \mathrm{z} 804.3$ and 986.4 , but no typical protein charge state distribution. The sample component could not be identified, but we presume it is a low-molecular-weight compound. Next, an aqueous solution of rhGH CRS $(1.5 \mathrm{mg} / \mathrm{mL})$ that was exposed to heat $\left(40{ }^{\circ} \mathrm{C}\right)$ for $24 \mathrm{~h}$ and then had been stored for 1 year at $-18^{\circ} \mathrm{C}$ was analyzed using the PB-PVS CE-TOF-MS system. The resulting base peak electropherogram (BPE) shows that several degradation products have been formed (Fig. 1c). As these products were not detected during the analysis of the CRS compound, we conclude that the applied CE-MS conditions do not cause interfering artifacts. That is, the observed extra compounds originate from heat exposure and/ or prolonged storage. The unknown impurity was again observed at $10.2 \mathrm{~min}$. The protein mass spectra obtained for the peaks between 11.5 and 13.0 min are depicted in Fig. 1d,
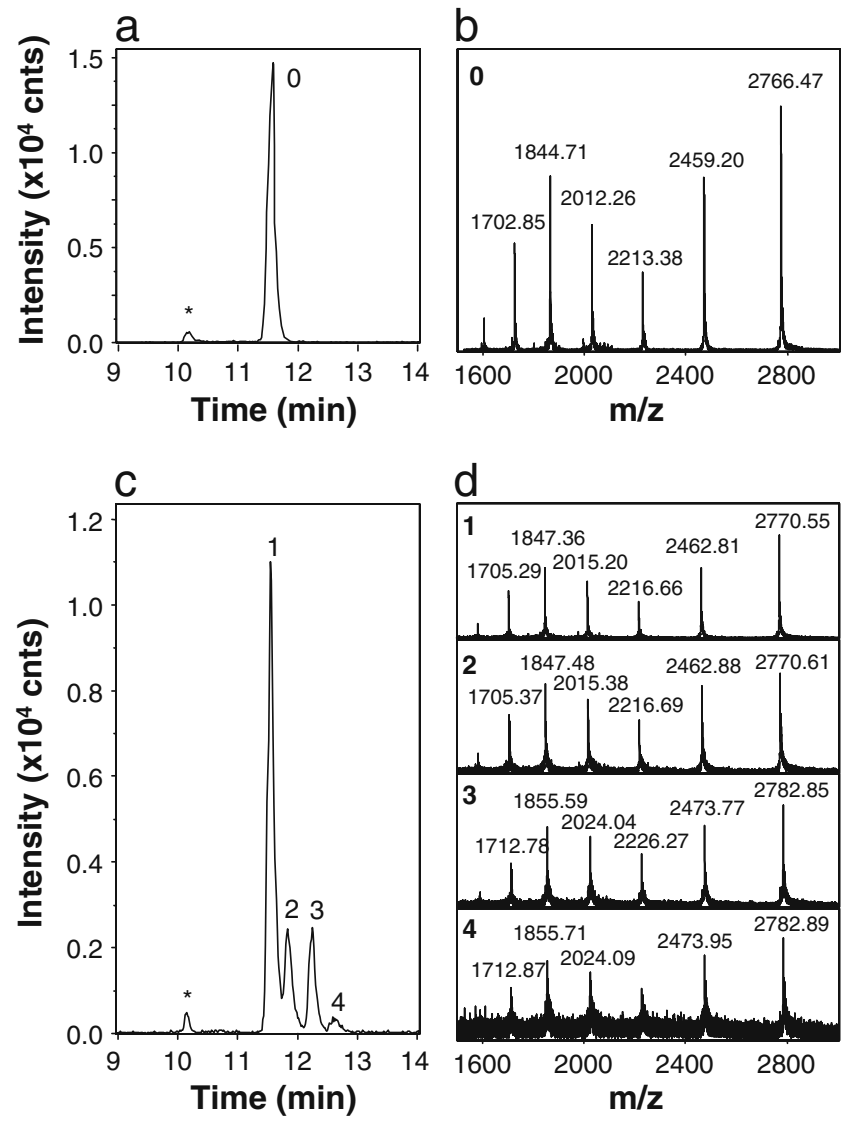

Fig. 1 CE-TOF-MS of $\mathbf{a}, \mathbf{b}$ rhGH CRS and $\mathbf{c}, \mathbf{d}$ heat-exposed and stored rhGH CRS using a PB-PVS coated capillary. a, c BPE $(\mathrm{m} / \mathrm{z} 1,500$ 3,000 ), the asterisk indicates an unknown non-proteinaceous unknown compound. b, $\mathbf{d}$ Mass spectra obtained in the apex of the indicated peaks. Conditions: BGE, 75 ammonium formate $(\mathrm{pH} 8.5)$; sheath liquid, acetonitrile-water-formic acid $(75 / 25 / 5, v / v / v)$ at $4 \mu \mathrm{L} / \mathrm{min}$. Sample preparation and other conditions, see Materials and methods section

yielding deconvoluted masses of 22156.1, 22157.0, 22252.3, and 22253.3 Da for peaks 1 to 4, respectively. Remarkably, no unmodified rhGH (22124 Da) was found in the sample, although the main degradation product (peak 1) had virtually the same migration time as rhGH CRS (11.6 min). The observed mass difference of 32 Da for peak 1 with respect to rhGH CRS suggests the main degradation product to be double oxidized rhGH. Oxidation of methionine residues is a common modification observed for rhGH [37, 38]. The gain of two oxygen atoms will hardly affect the electrophoretic mobility of the protein, as the oxidation induces no change in the protein charge and only a very small relative change in protein mass. The compound migrating at $11.8 \mathrm{~min}$ (peak 2) is clearly separated from the oxidized rhGH (peak 1) and differs only by $0.9 \mathrm{Da}$ in mass. Most probably, peak 2 is caused by rhGH that is oxidized (twice) as well as deamidated (once). Deamidation is a common protein degradation [39] that results in a small change of protein molecular mass $(+0.984 \mathrm{Da})$, but, at $\mathrm{pH} 8.5$, also in a net 
increase of one negative charge of the protein due to the formation of an aspartic acid residue. As a consequence, the protein's electrophoretic mobility changes significantly. The molecular mass of the degradation product migrating at 12.2 min (peak 3) is 128 Da higher than rhGH CRS. As this compound migrates after the deamidated product (peak 2), it most likely has gained at least two negative charges with respect to rhGH. Therefore, the mass difference of $128 \mathrm{Da}$ could be explained by the formation of two sulfonic acid groups from a protein disulfide bridge in addition to the double methionine oxidation described above. This would explain the gain of negative charge (two sulfonate groups) and molecular mass (eight oxygen atoms) leading to the observed electrophoretic mobility and molecular weight. Disulfide bridge breakage and oxidation has been described for bovine growth hormone [40], but to our knowledge, not for rhGH. Considering its molecular mass (22253.3 Da) and migration time (12.5 $\mathrm{min})$, the component causing peak 4 most probably is the singly deamidated form of the sulfonated/ oxidized product (peak 3). So, from the CE-TOF-MS results, it can be concluded that exposure of rhGH CRS in water to mild heat followed by prolonged storage can lead to oxidation of the protein accompanied by deamidation.

From our present results and those reported previously $[10,11]$, we can conclude that our CE-MS method with PBPVS coated capillaries is beneficial as it provides faster and more efficient and reproducible separations of rhGH than the $\mathrm{Ph}$. Eur. method, which employs bare fused-silica capillaries. In addition, the use of TOF-MS detection adds important selectivity, which enhances the reliability of the method providing additional separation. For example, peaks 1 and 3 in Fig. 1c would erroneously have been assigned to unmodified rhGH and a bisdeamidated product, respectively, when using CE-UV method of the Ph. Eur.

\section{Oxytocin}

Oxytocin is a nonapeptide hormone drug containing an internal disulfide bridge, which is used to induce or stimulate labor and to prevent post-partum hemorrhage. It was demonstrated that oxytocin is susceptible to $\mathrm{pH}$ dependent degradation at elevated temperatures [41]. Oxytocin itself has been analyzed before with CE-UV [42] and CE-MS [43], however, CE-MS characterization of degradation products has not been reported. Here, we investigated the possibility of CE-TOF-MS to reveal the composition of heat-stressed samples of oxytocin. Oxytocin solutions of $\mathrm{pH} 2.0,4.5,7.0$, and 9.0 that had been exposed to $70^{\circ} \mathrm{C}$ for $64 \mathrm{~h}$ were available for analysis. Oxytocin is a basic peptide ( $p I$ 8.7), therefore, a PB-DS-PB capillary coating was selected for CE-MS in combination with a lowpH BGE. Under these conditions, the peptide and capillary wall will be positively charged, and analyte adsorption will be minimized. DS was used instead of PVS to prepare the triple layer coating because it has been demonstrated that DS provides superior coating stability and separation efficiencies when applied as "in-between" polymeric layer for peptide and protein analysis [44].

Separation conditions were optimized using the heatexposed solution of oxytocin $(50 \mu \mathrm{g} / \mathrm{mL})$ at $\mathrm{pH} 2.0$. With a BGE of $50 \mathrm{mM}$ acetic acid ( $\mathrm{pH}$ 3.0), the BPE obtained with CE-MS revealed one main peak with a plate number of ca. 100,000. The mass spectrum of this peak comprised of distinct signals at several $\mathrm{m} / \mathrm{z}$ values, with the most intense at $\mathrm{m} / z$ 1007.43, which agrees well with the mass of protonated oxytocin. In order to optimize the CE performance, the concentration acetic acid of the BGE (pH 3.0) was varied between 50 and $1,000 \mathrm{mM}$. The plate number of oxytocin increased with increasing BGE concentration reaching an optimum $(300,000)$ at $525 \mathrm{mM}$ acetic acid. Moreover, with increasing BGE concentrations, the $\mathrm{CE}$ resolution improved, revealing additional, partially separated peaks. Acetic acid concentrations above $525 \mathrm{mM}$ did not result in higher plate numbers or better separation. Variation of the $\mathrm{pH}$ (3.0-5.0) of a BGE of $525 \mathrm{mM}$ acetic acid did not result in improved separation. Acetic acid at $525 \mathrm{mM}$ $(\mathrm{pH}$ 3.0) was then selected as BGE for further analysis. Under these conditions, migration time RSDs for oxytocin were less than $1.4 \% \quad(n=5)$, demonstrating the good performance of the PB-DS-PB CE-TOF-MS system. No additional peaks were observed indicating that the acidic BGE did not cause oxytocin degradation within the time frame of the CE analysis.

The BPE obtained upon CE-MS analysis of oxytocin degraded at $\mathrm{pH} 2.0$ showed five partially separated peaks. The mass spectrum of the main peak $(8.0 \mathrm{~min})$ was dominated by an intense signal at $m / z$ 1007.43, whereas the mass spectra of the degradation products migrating at $6.8 \mathrm{~min}$ and between 7.0-7.6 min, showed most abundant signals at $\mathrm{m} / \mathrm{z} 1009.39$ and 1008.41 , respectively. Using these masses, extracted ion electropherograms (EIEs) were constructed (Fig. 2a). The EIE of $m / z 1007.43$ represents the unmodified oxytocin (single peak), whereas the EIE of $m / z 1008.41$ exhibits three peaks. Considering the migration times of these three degradation products with respect to oxytocin, they probably are less positively charged than oxytocin. In combination with the gain in mass of about 1 Da compared with oxytocin, one can conclude that these degradation products most likely are the result of single deamidations of oxytocin. Indeed, oxytocin contains three possible deamidation sites and apparently the three monodeamidated oxytocins can be separated by CE. The EIE of $\mathrm{m} / z 1009.39$ reveals the presence of bisdeamidated oxytocin (Fig. 2a). As the peak shows an unresolved shoulder, we concluded that at least two bisdeamidated variants have been formed during heat degradation. 

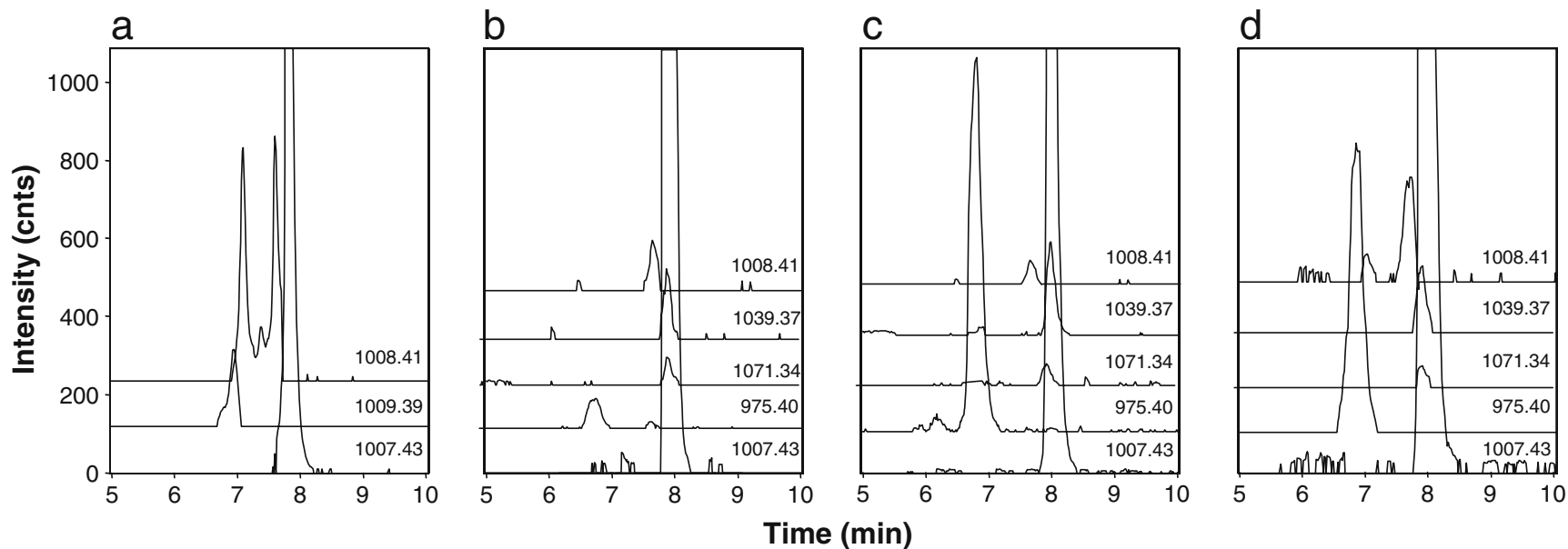

Fig. 2 CE-TOF-MS of heat-exposed oxytocin solutions of a $\mathrm{pH} 2.0$, b $\mathrm{pH} 4.5$, c pH 7.0, and $\mathbf{d ~ p H ~} 9.0$ using a PB-DS-PB coated capillary. EIEs are at indicated $\mathrm{m} / \mathrm{z}$ values $( \pm 0.01 \mathrm{Da})$. Conditions: BGE,

Oxytocin samples that were solved in phosphate buffer of $\mathrm{pH} 4.5,7.0$, and 9.0 and exposed to $70^{\circ} \mathrm{C}$ for $64 \mathrm{~h}$ were also analyzed with CE-MS, showing several degradation products (Fig. 2b-d; Table 1). Clearly, deamidation is most predominant at $\mathrm{pH} 2.0$; the $\mathrm{pH}$ 4.5-9.0 samples show only minor mono-deamidation and no bisdeamidation. Interestingly, these samples show two degradation products which comigrate with oxytocin. The mass increase of 31.95 and $63.91 \mathrm{Da}$ with respect to oxytocin indicates that these two products most likely are the result of the addition of one and two sulfur atoms to their molecular structure. These modifications do not induce a change of molecular charge and therefore most probably are not separated. Although unusual, trisulfide formation as a result of a $\beta$-elimination processes caused by heat exposure has been reported [45]. The samples dissolved in buffer of $\mathrm{pH} 7.0$ and 9.0 showed another significant degradation product with $\mathrm{m} / \mathrm{z} 975.40$ at a migration time of $6.8 \mathrm{~min}$. From the isotope pattern, it can be concluded that the compound is doubly protonated and thus has a molecular weight of $1,948.8 \mathrm{Da}$. This gain in mass with respect to oxytocin suggests the product to be the result of a dimerization process involving loss of two
$525 \mathrm{mM}$ acetic acid (pH 3.0); sheath liquid, isopropanol-water-acetic acid $(75 / 25 / 0.1, v / v / v)$ at $2 \mu \mathrm{L} / \mathrm{min}$. Sample preparation and other conditions, see Materials and methods section

sulfur groups [41]. The exact mechanism of the formation of this product is unclear.

Not much detailed information on oxytocin degradation in therapeutic formulations is available in literature. Oxytocin purity is generally assessed using liquid chromatography (LC) with UV detection [46, 47], taking the method described in the $\mathrm{Ph}$. Eur. [48] as a starting point. However, these methods do not allow identification of the degradation products. Just recently, application of reversed-phase (RP)LC-MS was reported for the assignment of oxytocin degradation products [41]. With CETOF-MS, we observed the same degradation products as with the RPLC-MS method. The CE-MS analysis time was considerably shorter (10 vs. $40 \mathrm{~min}$ ); however, in contrast to CE, RPLC allowed separation of degradation products containing sulfur modifications such as tri- and tetrasulfide bridges.

Recombinant human interferon- $\beta$-1a

The drug rhIFN- $\beta$ is a $23-\mathrm{kDa} N$-glycosylated protein $\left(\mathrm{Asn}^{80}\right)$, which is used for treatment of multiple sclerosis.

Table 1 Compounds observed with CE-TOF-MS in heat-exposed oxytocin solutions of various $\mathrm{pH}$

\begin{tabular}{|c|c|c|c|c|c|}
\hline \multirow[t]{2}{*}{$\mathrm{m} / \mathrm{z}$ (charge) } & \multirow[t]{2}{*}{ Compound } & \multicolumn{4}{|c|}{ Relative peak area $(\%)$} \\
\hline & & $\mathrm{pH} 2.0$ & $\mathrm{pH} 4.5$ & $\mathrm{pH} 7.0$ & $\mathrm{pH} 9.0$ \\
\hline $975.40(2+)$ & Oxytocin "dimer" & ND & 2.4 & 14.5 & 9.7 \\
\hline $1007.43(1+)$ & Oxytocin & 60.6 & 90.6 & 75.8 & 80.3 \\
\hline $1008.41(1+)$ & Deamidated oxytocin & 31.4 & 1.4 & 1.1 & 2.8 \\
\hline $1009.39(1+)$ & Bisdeamidated oxytocin & 8.0 & ND & ND & ND \\
\hline $1039.37(1+)$ & "Trisulfide" oxytocin & ND & 4.8 & 7.0 & 5.7 \\
\hline $1071.34(1+)$ & "Tetrasulfide" oxytocin & ND & 0.9 & 1.7 & 1.4 \\
\hline
\end{tabular}

$N D$ not detected 

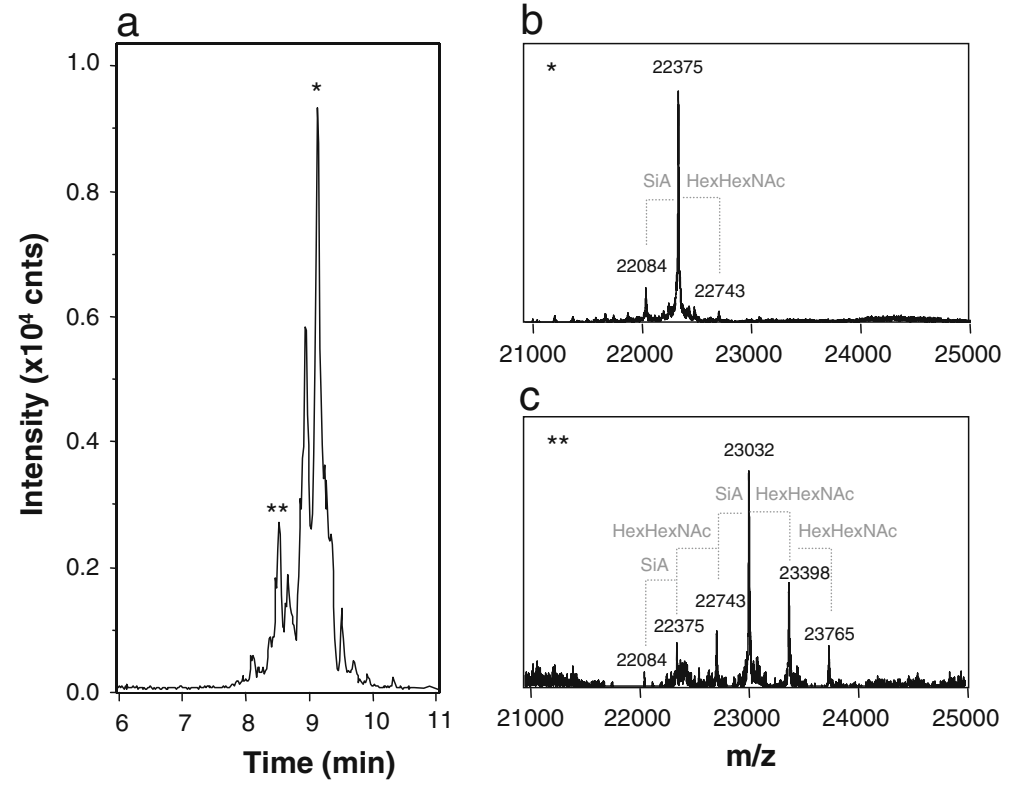

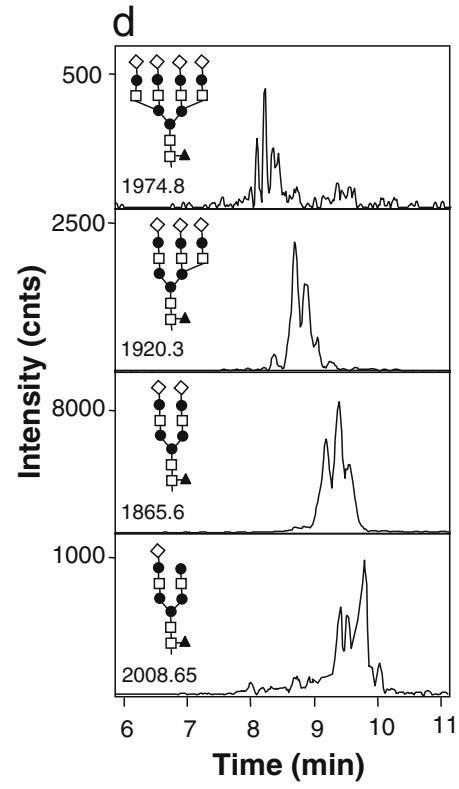

Fig. 3 CE-TOF-MS of rhIFN- $\beta$ using a PB-DS-PB coated capillary. a BPE $(m / z 1,500-3,000)$. Deconvoluted mass spectrum obtained in the apex of the peak migrating $\mathbf{b}$ at $9.1 \mathrm{~min}$ (asterisk) and $\mathbf{c}$ at $8.5 \mathrm{~min}$ (double asterisks). d EIEs are at indicated $\mathrm{m} / \mathrm{z}$ values $( \pm 0.2 \mathrm{Da})$; assigned glycan structures are shown as insets. Symbols: closed circle,

Over the past years, biosimilars of rhIFN- $\beta$ have been introduced onto the market. It has been demonstrated that the glycosylation of rhIFN- $\beta$ may significantly affect its biological activity [49]. Therefore, establishment of the glycoform heterogeneity among batches of rhIFN- $\beta$ can be of great importance. Moreover, glycoform profiling can be a tool for characterizing and comparing rhIFN- $\beta$ products, including biosimilars. As protein glycosylation affects charge and size of proteins, CE - with its charge-to-mass separation mechanism - can be very useful for glycoprotein profiling. We studied whether rhIFN- $\beta$ can be analyzed hexose; closed upright triangle, fucose; open square, $N$-acetylhexosamine; open diamond, sialic acid. Conditions: BGE, $50 \mathrm{mM}$ acetic acid ( $\mathrm{pH} 3.0)$; sheath liquid, isopropanol-water-acetic acid (25/75/0.5, $v / v / v)$ at $2 \mu \mathrm{L} / \mathrm{min}$. Sample preparation and other conditions, see Materials and methods section

with our CE-TOF-MS system and the information that can be derived from the obtained data.

rhIFN- $\beta$ is a basic protein ( $p I$ 9.6) and, therefore, to avoid protein adsorption to the capillary wall, a PB-DS-PB coated capillary was selected in combination with an acidic BGE. Previously, we found a $50-\mathrm{mM}$ acetic acid BGE (pH 3.0) to be optimal for basic proteins [32]. The same BGE appeared to provide good separation of $\operatorname{rhIFN}-\beta$ glycoforms. The composition of the sheath liquid was briefly optimized. Selection of an isopropanol content of $25 \%$ and a relatively high acetic acid concentration $(0.5 \%)$

Table 2 Molecular mass, glycan composition, relative peak area, and migration time for rhIFN- $\beta$ glycoforms as observed with CE-TOF-MS

\begin{tabular}{|c|c|c|c|c|c|c|c|c|c|c|}
\hline Deconvoluted mass & 22084 & 22375 & 22743 & 23032 & 23105 & 23398 & 23692 & 23765 & 24054 & 24424 \\
\hline Glycan $^{\mathrm{a}}$ & 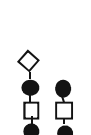 & 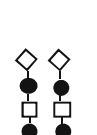 & SY & & & & & & 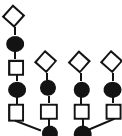 & 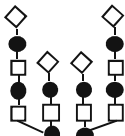 \\
\hline Relative peak area $(\%)^{b}$ & 6.3 & 52.3 & 6.7 & 12.0 & 5.5 & 9.3 & 1.8 & 4.2 & 1.3 & 0.6 \\
\hline Migration time $(\mathrm{min})^{\mathrm{c}}$ & 9.5 & 9.1 & 8.9 & 8.5 & 8.6 & 8.5 & 8.1 & 8.3 & 8.0 & 7.9 \\
\hline
\end{tabular}

${ }^{a}$ Symbols: closed circle, hexose; closed upright triangle, fucose; open square, $N$-acetylhexosamine; open diamond, sialic acid

${ }^{\mathrm{b}}$ Total area of peaks observed in the respective EIE

${ }^{\mathrm{c}}$ Migration time of the most intense peak observed in the respective EIE 
appeared to be important to achieve good signals for rhIFN$\beta$. So, a sheath liquid of isopropanol-water-acetic acid (25/ $75 / 0.5, v / v / v)$ at $2 \mu \mathrm{L} / \mathrm{min}$ was applied. Typical migration time RSDs for rhIFN- $\beta$ under the selected conditions were less than $1.6 \%(n=5)$.

The BPE obtained after CE-MS of rhIFN- $\beta(450 \mu \mathrm{g} / \mathrm{mL})$ shows a distinct pattern of partially resolved bands (i.e., glycoforms) between 7.8 and 10.0 min (Fig. 3a). Figure 3b shows the deconvoluted mass spectrum obtained in the apex of the most intense peak $(9.2 \mathrm{~min})$, indicating a molecular mass of 22,375 Da. Some minor signals of other glycoforms with masses of 22,082 and 22,743 Da were also observed in the spectrum. Deconvoluted spectra of other peaks in the BPE also showed multiple compounds. For example, the deconvoluted spectrum obtained for the peak at $8.5 \mathrm{~min}$ shows at least five proteinaceous compounds (Fig. 3c). In order to obtain a full picture of the number of different glycoforms, deconvoluted mass spectra were constructed with 0.1 -min intervals in the range of 7.8 to $10.0 \mathrm{~min}$. From these mass spectra, a total of ten distinctive masses (Table 2) representing different glycoforms could be determined; unglycosylated rhIFN- $\beta$ (20027 Da) was not detected. To assign the glycoforms, mass differences between the ten glycoforms were determined. Merely differences of 291 and $365 \mathrm{Da}$ were found, indicating the loss or gain of either a sialic acid unit or a hexose $\mathrm{N}$-acetylhexosamine (HexHexNAc) unit, respectively (Fig. 3b, c). In addition, it has been reported that the major glycoform rhIFN- $\beta$ is a fucosylated disialylated biantennary structure with four hexose and five $N$-acetylhexosamine units $[49,50]$. This glycoform, including the amino acid chain of $\operatorname{rhIFN}-\beta$, has a molecular weight of 22,375 Da. Thus, based on this glycan structure and the calculated mass differences, the most probable glycan composition of the other nine glycoforms were derived (Table 2). We observe five out of the six glycoforms described in the Ph. Eur. monograph of IFN- $\beta$ [51]. The nonsialylated isoform was not present in the analyzed sample. On the other hand, our CE-MS method revealed additional low-abundance glycoforms with more sialic acid and HexHexNAc units than described in the $\mathrm{Ph}$. Eur. Our results also match well with a previous report on ESI-MS of rhIFN- $\beta$ [49], which reported six out of the ten glycoforms we detected. This underlines the importance of an efficient separation prior to MS analysis in order to prevent signal suppression and allow reliable and more detailed assignment.

For each assigned glycoform, an EIE was constructed using the most abundant $\mathrm{m} / \mathrm{z}$ value of the glycoform's respective charge envelope. Four selected EIEs are depicted in Fig. 3d. From the EIEs, relative peak areas for all ten detected glycoforms were obtained leading to a quantitative glycoform distribution, assuming an equimolar detector response per glycoform (Table 2). The glyco- form with the lowest abundance was almost $1 \%$ in area with respect to the most abundant isoform, but could still be detected reliably. Relative abundance of glycoforms may show useful for in-process and quality control of rhIFN- $\beta$ batches or to compare biosimilar products. From the EIEs, it is clear that the overall migration time of the glycoforms decreases with the degree of sialytion (Fig. 3d). Differences in the number of HexHexNAc groups present in a glycoform with the same number of sialic acid groups only lead to a slight change of migration time (Table 2). These observations are in good agreement with results reported for CE-MS of other glycoproteins $[12,52,53]$. It should be noted that, for each glycoform, no well-defined peak but rather a reproducible pattern of partially separated compounds was observed (Fig. 3d). Degradation of sialic acid groups, which theoretically can occur at low $\mathrm{pH}$, is most unlikely during $\mathrm{CE}$ analysis as this would require much harsher conditions $\left(\mathrm{pH} 2.0 ; 80^{\circ} \mathrm{C}\right)$ and reaction times $(>1 \mathrm{~h})$ than applied $\left(\mathrm{pH} 3.0 ; 20^{\circ} \mathrm{C}\right.$; and $\left.<10 \mathrm{~min}\right)$ [54]. Moreover, the obtained glycoform profile was highly repeatable. Therefore, we speculate the peak clusters to be glycan isomers, as also reported in the $\mathrm{Ph}$. Eur. monograph [51], with slightly different electrophoretic mobilities.

\section{Concluding remarks}

CE-TOF-MS methods applying on noncovalently coated capillaries have been evaluated for biopharmaceutical analysis. The acidic protein rhGH could be characterized using a PB-PVS capillary coating and a medium-pH BGE, whereas the PB-DS-PB coated capillary in combination with a low-pH BGE appeared to be very suitable for the analysis of the basic pharmaceuticals oxytocin and rhIFN$\beta$. Highly similar and/or related degradation products and glycoforms could be assigned by the combination of $\mathrm{CE}$ with TOF-MS. From the presented results, it can be concluded that $\mathrm{CE}$ is especially suitable for the separation of protein modifications leading to charge differences. For example, degradation products as result of deamidation or sulfonic acid formation were efficiently resolved from the parent compound. Also, protein glycoforms which differ in number of sialic acid groups could be separated. In case that modifications did not affect the overall protein charge, the high mass resolution and accuracy of the TOF-MS still allowed assignment of components. So, we believe there is strong potential for CE-TOF-MS systems using noncovalent coated capillaries for the purity and stability analysis of biopharmaceuticals. Moreover, the CE-MS systems seem very useful for highly specific glycoform profiling. Currently, we are studying the usefulness of CE-TOF-MS systems for the characterization and comparison of biosimilars. 
Acknowledgment This research was supported by the Dutch Technology Foundation STW, Applied Science Division of NWO, and the Technology Program of the Ministry of Economic Affairs

Open Access This article is distributed under the terms of the Creative Commons Attribution Noncommercial License which permits any noncommercial use, distribution, and reproduction in any medium, provided the original author(s) and source are credited.

\section{References}

1. Pharmaceutical Research and Manufacturers of America, http:// www.phrma.org/sites/phrma.org/files/attachments/NDA 2009. pdf, last accessed Sept. 21, 2010

2. Pharmaceutical Research and Manufacturers of America, http:// www.phrma.org/sites/phrma.org/files/attachments/Biotech\% 202008.pdf, last accessed Sept. 21, 2010

3. Sharma B (2007) Biotechnol Adv 25:310-317

4. Schellekens H (2009) Drug Disc Today 14:495-499

5. Haselberg R, de Jong GJ, Somsen GW (2007) J Chromatogr A 1159:81-109

6. Haselberg R, de Jong GJ, Somsen GW (2010) Electrophoresis in press

7. Brenner-Weiss G, Kirschhofer F, Kuhl B, Nusser M, Obst U (2003) J Chromatogr A 1009:147-153

8. Liang Z, Yang Q, Zhang W, Zhang L, Zhang Y (2003) Chromatographia 57:617-621

9. Staub A, Giraud S, Saugy M, Rudaz S, Veuthey JL, Schappler J (2010) Electrophoresis 31:388-395

10. Catai JR, Toraño JS, De Jong GJ, Somsen GW (2007) Analyst $132: 75-81$

11. Catai JR (2007) Sastre Toraño J, Jongen PMJM, de Jong GJ, Somsen GW. J Chromatogr B 852:160-166

12. Neusüß C, Demelbauer U, Pelzing M (2005) Electrophoresis 26:1442-1450

13. Giménez E, Benavente F, Barbosa J, Sanz-Nebot V (2008) Electrophoresis 29:2161-2170

14. Balaguer E, Neusüß C (2006) Anal Chem 78:5384-5393

15. Balaguer E, Neusüß C (2006) Chromatographia 64:351-357

16. Balaguer E, Demelbauer U, Pelzing M, Sanz-Nebot V, Barbosa J, Neusüß C (2006) Electrophoresis 27:2638-2650

17. Lucy CA, MacDonald AM, Gulcev MD (2008) J Chromatogr A 1184:81-105

18. Stutz H (2009) Electrophoresis 30:2032-2061

19. Huhn C, Ramautar R, Wuhrer M, Somsen GW (2010) Anal Bioanal Chem 396:297-314

20. Katayama H, Ishihama Y, Asakawa N (1998) Anal Chem 70:5272-5277

21. Katayama H, Ishihama Y, Asakawa N (1998) Anal Chem 70:2254-2260

22. Weinbauer M, Stutz H (2010) Electrophoresis 31:1805-1812

23. Robb CS (2007) J Liq Chromatogr Rel Technol 30:729-759

24. Nehmé R, Perrin C, Cottet H, Blanchin MD, Fabre H (2009) Electrophoresis 30:1888-1898

25. Nehmé R, Perrin C, Cottet H, Blanchin MD, Fabre H (2008) Electrophoresis 29:3013-3023

26. Kitagawa F, Kamiya M, Otsuka K (2008) J Chromatogr B $875: 323-328$
27. Kitagawa F, Kamiya M, Okamoto Y, Taji H, Onoue S, Tsuda Y, Otsuka K (2006) Anal Bioanal Chem 386:594-601

28. Danger G, Ramonda M, Cottet H (2007) Electrophoresis 28:925931

29. Boonsong K, Caulum MM, Dressen BM, Chailapakul O, Cropek DM, Henry CS (2008) Electrophoresis 29:3128-3134

30. Catai JR, Toraño JS, de Jong GJ, Somsen GW (2006) Electrophoresis 27:2091-2099

31. Catai JR, Tervahauta HA, De Jong GJ, Somsen GW (2005) J Chromatogr A 1083:185-192

32. Haselberg R, de Jong GJ, Somsen GW (2010) Anal Chim Acta 678:128-134

33. Haselberg R, de Jong GJ, Somsen GW (2009) J Sep Sci 32:24082415

34. Haselberg R, Van Der Sneppen L, Ariese F, Ubachs W, Gooijer C, De Jong GJ, Somsen GW (2009) Anal Chem 81:1017210178

35. European Pharmacopoeia, Supplement 6.8, Somatropin for Injection (sixth ed.), Council of Europe, Strasbourg (2010) pp. 2935-2937

36. Yan X, Watson W (2004) Shing Ho P, Deinzer ML. Mol Cell Proteomics 3:10-23

37. Karlsson G, Gellerfors P, Persson A, Norén B, Edlund PO, Sandberg C, Birnbaum S (1999) J Chromatogr A 855:147-155

38. Hepner F, Cszasar E, Roitinger E, Lubec G (2005) Proteome Sci $3: 1-12$

39. Manning MC, Chou DK, Murphy BM, Payne RW, Katayama DS (2010) Pharm Res 27:544-575

40. Miller BL, Hageman MJ, Thamann TJ, Barron LB, Schoneich C (2003) J Pharm Sci 92:1698-1709

41. Hawe A, Poole R, Romeijn S, Kasper P, Van Der Heijden R, Jiskoot W (2009) Pharm Res 26:1679-1688

42. Solínová V, Kasicka V, Koval D, Barth T, Ciencialová A, Záková L (2004) J Chromatogr B 808:75-82

43. Sanz-Nebot V, Benavente F, Balaguer E, Barbosa J (2003) Electrophoresis 24:883-891

44. Nehmé R, Perrin C, Cottet H, Blanchin MD, Fabre H (2008) Electrophoresis:3013-3023

45. Windisch V, DeLuccia F, Duhau L, Herman F, Mencel JJ, Tang SY, Vuilhorgne M (1997) J Pharm Sci 86:359-364

46. Ashenafi D, Van Hemelrijck E, Chopra S, Hoogmartens J, Adams E (2010) J Pharm Biomed Anal 51:24-29

47. Chaibva FA, Walker RB (2007) J Pharm Biomed Anal 43:179185

48. European Pharmacopoeia, Supplement 6.8, Oxytocin (sixth ed.), Council of Europe, Strasbourg (2010) pp. 2593-2594

49. Orru S, Amoresano A, Siciliano R, Napoleoni R, Finocchiaro O, Datola A, De Luca E, Sirna A, Pucci P (2000) Biol Chem 381:717

50. Dissing-Olesen L, Thaysen-Andersen M, Meldgaard M, Hojrup P, Finsen B (2008) J Pharmacol Exp Ther 326:338-347

51. European Pharmacopoeia, Supplement 6.8, Interferon beta-1a concentrated solution (sixth ed.), Council of Europe, Strasbourg (2010) pp. 4904-4907

52. Thakur D, Rejtar T, Karger BL, Washburn NJ, Bosques CJ, Gunay NS, Shriver Z, Venkataraman G (2009) Anal Chem 81:8900-8907

53. Sanz-Nebot V, Balaguer E, Benavente F, Neusüß C, Barbosa J (2007) Electrophoresis 28:1949-1957

54. Fernando SF, Woonton BW (2010) J Food Comp Anal 23:359366 